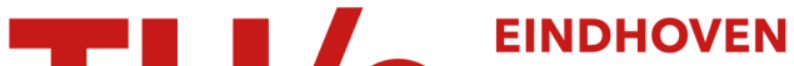 \\ UNIVERSITY OF \\ TECHNOLOGY
}

\section{High-resolution angle-of-arrival measurement of the mobile radio channel}

Citation for published version (APA):

Jong, de, Y. L. C., \& Herben, M. H. A. J. (1999). High-resolution angle-of-arrival measurement of the mobile radio channel. IEEE Transactions on Antennas and Propagation, 47(11), 1677-1687.

https://doi.org/10.1109/8.814947

DOI:

10.1109/8.814947

Document status and date:

Published: 01/01/1999

Document Version:

Publisher's PDF, also known as Version of Record (includes final page, issue and volume numbers)

Please check the document version of this publication:

- A submitted manuscript is the version of the article upon submission and before peer-review. There can be important differences between the submitted version and the official published version of record. People interested in the research are advised to contact the author for the final version of the publication, or visit the $\mathrm{DOI}$ to the publisher's website.

- The final author version and the galley proof are versions of the publication after peer review.

- The final published version features the final layout of the paper including the volume, issue and page numbers.

Link to publication

\section{General rights}

Copyright and moral rights for the publications made accessible in the public portal are retained by the authors and/or other copyright owners and it is a condition of accessing publications that users recognise and abide by the legal requirements associated with these rights.

- Users may download and print one copy of any publication from the public portal for the purpose of private study or research.

- You may not further distribute the material or use it for any profit-making activity or commercial gain

- You may freely distribute the URL identifying the publication in the public portal.

If the publication is distributed under the terms of Article $25 \mathrm{fa}$ of the Dutch Copyright Act, indicated by the "Taverne" license above, please follow below link for the End User Agreement:

www.tue.nl/taverne

Take down policy

If you believe that this document breaches copyright please contact us at:

openaccess@tue.nl

providing details and we will investigate your claim. 


\title{
High-Resolution Angle-of-Arrival Measurement of the Mobile Radio Channel
}

\author{
Yvo L.C. de Jong, Student Member, IEEE, and Matti H. A. J. Herben, Senior Member, IEEE
}

\begin{abstract}
The accurate planning of microcellular mobile radio networks requires a thorough understanding of the mechanisms that govern radio wave propagation in urban environments. Combined measurements of time delays and angles-of-arrival (AoA's), both in azimuth and elevation, of individual multipath contributions are expected to be very helpful in the validation and improvement of existing ray-tracing propagation models. To this end, we have applied the uniform circular array (UCA)MUSIC angular superresolution algorithm to complex impulse response measurement data obtained from a synthetic uniform circular array. Beamspace processing and forward/backward averaging were applied to cope with strongly correlated signals. The presented experimental results show that our approach is capable of identifying the dominant multipaths in actual mobile propagation environments.
\end{abstract}

Index Terms - Delay estimation, direction of arrival estimation, land mobile radio, mobile communication.

\section{INTRODUCTION}

A $\mathrm{S}$ a result of today's rapidly growing mobile telecommunications market, mobile network operators are considering various methods to increase the capacity of their networks. Probably the most promising development in this respect is the deployment of microcells in urban areas where very high traffic intensities are expected. As is well known, ultrahigh frequency (UHF) radio wave propagation in urban environments is typically a multipath phenomenon, i.e., signals travel between the transmitter and the receiver following discrete paths. In microcells, the base stations (BS) are usually mounted below the rooftop level and the line-of-sight (LOS) propagation path is often blocked. Then propagation via reflection, diffraction, and scattering from various objects in the environment becomes significant.

For the planning of macrocells, network planners have traditionally been relying on the characterization of the mobile radio channel in terms of various statistical parameters, obtained from extensive field strength measurements. In microcellular areas, however, the propagation strongly depends on local features of the environment (such as locations, shapes and dielectric properties of the buildings) and important channel characteristics such as path loss and delay spread become very location-specific. Deterministic propagation prediction models, capable of providing location-specific field predictions on the basis of an accurate building database and physical models of

Manuscript received February 10, 1998; revised May 26, 1999. This work was supported by KPN Research, Leidschendam, The Netherlands.

The authors are with the Faculty of Electrical Engineering, Eindhoven University of Technology, 5600 MB Eindhoven, The Netherlands.

Publisher Item Identifier S 0018-926X(99)09946-9. the occurring propagation phenomena are, therefore, receiving increased attention. Result of this development is a growing need for a more fundamental understanding of the dominant propagation mechanisms in the mobile radio channel. For this purpose, a number of groups have been active in the measurement of channel characteristics at the level of the individual multipath components, in particular, by separating the multipath waves on the basis of their different propagation delay times and angles-of-arrival (AoA's) [1]-[6].

Delay measurements are usually performed in the time domain using the pseudonoise (PN) correlation approach [7], [8]. For the measurement of AoA's, both directional antennas [1] and (synthetic) phased arrays have been applied, the latter of which benefit from their relative ease of use and suitability for digital data processing schemes such as high-resolution algorithms. Synthetic arrays have the inherent advantages that mutual coupling between adjacent array elements is avoided, and that the radiation pattern of the elements is not affected. The most common array geometries used in practice are the uniform linear array (ULA) [5], the rectangular lattice [2], [3] and the uniform circular array (UCA) [4], [6]. A major drawback of the ULA is that it is not suited for twodimensional (2-D) (azimuth and elevation) AoA estimation. As has been pointed out in [2], the common assumption that all waves impinge from the horizontal plane leads to considerable errors in the azimuth estimation if the elevation differs from zero. As compared to the rectangular array, the UCA can be synthesized in a particularly simple fashion, by placing an antenna on a rotating arm. Furthermore, due to the rotational symmetry of the array configuration, the resolution performance is independent of the azimuth angles of the incident waves.

Thus far, superresolution estimation of multipath wave AoA's using UCA's has been disregarded in the literature due to the assumed low robustness of high-resolution algorithms to noise and their requirement of a priori knowledge of the number of incident waves [6]. Also, AoA estimation using UCA's has hitherto been limited to azimuth angles only. In this paper, we present a high-resolution approach toward 2-D (azimuth and elevation) AoA estimation which is suited for this array configuration. The increased resolution capability enables the identification of the dominant propagation paths contributing to the total received field strength, and a quantitative analysis of the propagation mechanisms involved.

The organization of the paper is as follows. Section II gives a description of the measurement equipment. In Section III, we introduce the data model and some notation that will be used in 


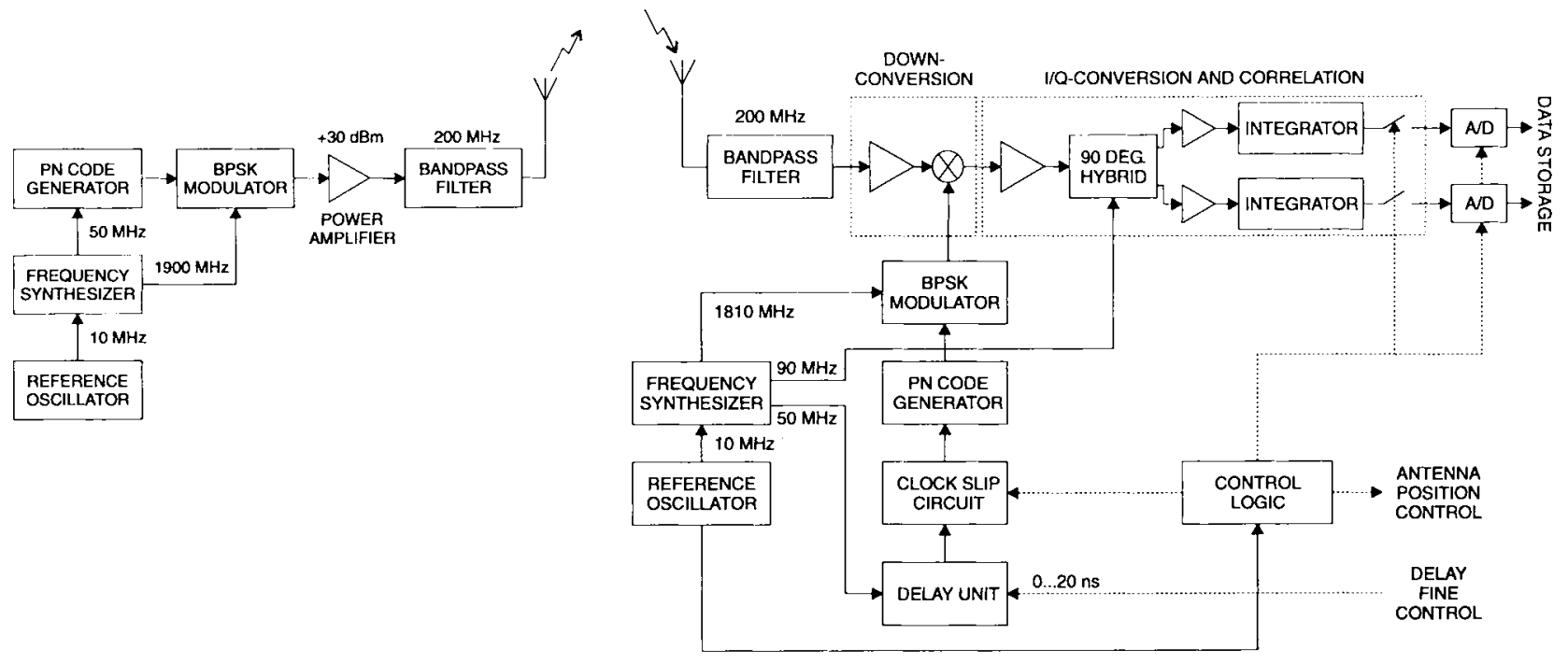

Fig. 1. Wide-band radio channel sounder. (a) Transmitter. (b) Receiver.

the rest of this paper. The algorithm for the accurate estimation of AoA's is described in Section IV, computer simulations of the angular resolution performance are given in Section V, and Section VI presents experimental results that were obtained in actual mobile propagation environments. Finally, conclusions are drawn in Section VII.

\section{MEASUREMENT SyStem}

\section{A. Channel Sounder}

The system used for our measurements is built up around a stepping-correlator radio channel sounder, which is based on the popular pseudonoise (PN) correlation method [7], [8]. This technique utilizes a periodic PN binary sequence and exploits its autocorrelation properties to provide an estimate of the complex impulse response (CIR) of the channel under measurement. A schematic diagram of the channel sounder is given in Fig. 1.

At the channel sounder transmitter, a $1900 \mathrm{MHz}$ carrier is phase-reversal modulated by a 50-Mbit/s maximum-length PN sequence with a period of 511 bits. The modulator output is amplified to a power of $+30 \mathrm{dBm}$, bandpass filtered to 200 $\mathrm{MHz}$, and, subsequently, radiated from a suitable antenna.

At the channel sounder receiver, the CIR of the radio channel is estimated by demodulating and correlating the received signal with a locally generated replica maximumlength sequence, which is time-shifted relative to the received signal in discrete steps of $20 \mathrm{~ns}$ by means of the clock slip circuit. Each output value is the result of an integration over 11 sequence periods, which yields an increased dynamic range. A single CIR sample is produced every $0.15 \mathrm{~ms}$, and the acquisition of a complete CIR consisting of 511 consecutive samples, takes $78.34 \mathrm{~ms}$. The measurement data samples are digitized, transferred to a personal computer and stored on a hard disk. The resulting measurement files contain CIR's of $10.22 \mu$ s duration, with an effective sampling interval of $20 \mathrm{~ns}$ and a dynamic range of $40 \mathrm{~dB}$. For quasicoherent measurements, the frequency synthesizers at both the transmitter and the receiver are locked to highly stable rubidium clocks (short-term stability better than $5 \times 10^{-11}$ ). To ensure accurate power density measurements, the channel sounder is calibrated prior to the measurements by connecting a fixed, known attenuation between the transmitter and the receiver.

Independent of the clock slip circuit, the timing difference between the transmitted and the locally generated PN sequences is controlled by a programmable delay unit, which is fixed during each measurement, but can be externally adjusted from 0 to $20 \mathrm{~ns}$ between measurements. As will become apparent later, this improves the robustness of our angular superresolution algorithm.

\section{B. Synthetic Array}

The antenna array is synthesized using a single antenna on a rotating arm which is positioned by a motor under control of the personal computer used for data acquisition. While the channel sounder is acquiring a prespecified number of channel responses, the antenna is rotated at constant speed along a horizontal circle. One cycle of the antenna is completed after $8.3 \mathrm{~s}$, which corresponds to the acquisition time of $M=106$ channel responses (Fig. 2). Under the assumption of channel stationarity during the measurement, this procedure is effectively identical to the simultaneous measurement of the radio channel CIR at the elements of a UCA of antennas.

The continuous movement of the rotating antenna introduces sensor positioning errors with respect to the assumed fixed positions $\gamma_{0}, \gamma_{1}, \cdots, \gamma_{M-1}$ of the UCA elements (view Fig. 2). These errors are dependent on the position $i(0 \leq i<511)$ of the considered delay instant within the measured CIR's and can be as large as $360^{\circ} / 106=3.4^{\circ}$, which is unacceptable for our purpose. They can, however, be completely compensated for by adding the correction angle $+(i / 511) \cdot 360^{\circ}$ to the 


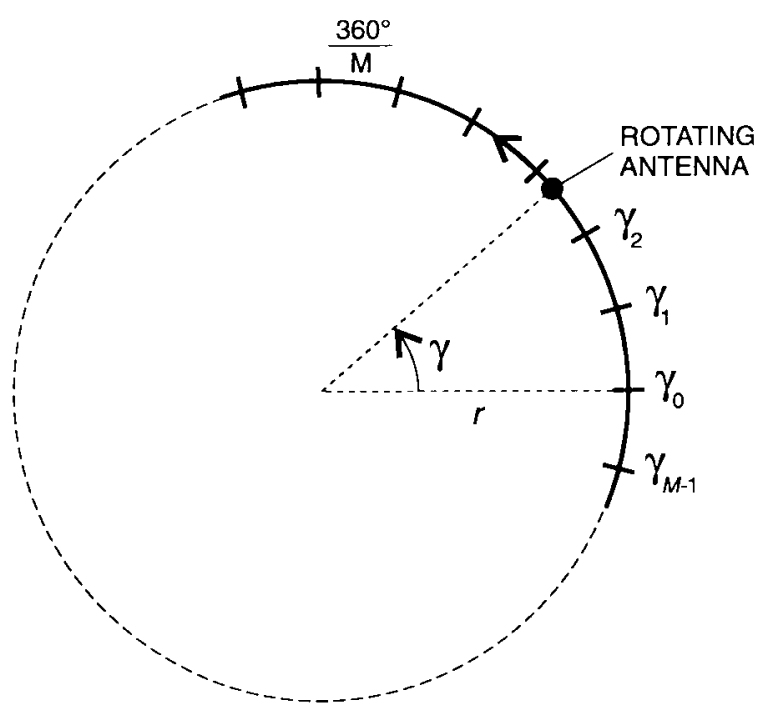

Fig. 2. Synthetic UCA.

estimated azimuth of each resolved multipath contribution associated with the $i$ th delay bin.

An important UCA parameter is its radius $r$. As the angular estimation accuracy and resolution capability decrease with decreasing array dimensions, it is important to choose the UCA radius sufficiently large. However, there are also some important factors that limit the array size to be employed.

1) Plane Wave Assumption: The assumption that the electromagnetic field around the array can be modeled as the superposition of plane waves, which is used by the angular superresolution algorithm, requires the array size to be small compared to the distance of the array to the nearest scattering center.

2) Narrow-Band Array Assumption: To ensure that each multipath wave is received identically at all array elements except for a phase factor, the array size should be small compared to the distance covered at the speed of light during a bit period. This distance is $6 \mathrm{~m}$ in the present case.

3) Practical Considerations: Practical requirements with respect to size and weight also put limitations on the UCA radius.

We have experimented with various array dimensions, and found a UCA radius of $30 \mathrm{~cm}$, which is approximately two times the wavelength, to be a good compromise between the above factors.

Although the oscillators at the transmitter and receiver are locked to very precise frequency standards due to small frequency differences there is an inevitable drift of the measured phase that must be corrected in postprocessing. To this end, the channel response is measured during two consecutive cycles of the rotating antenna and the phase drift is estimated from the average phase difference $\Delta \phi_{d}$ between the second and the first cycle. The measured phase drift is then corrected by multiplication of the array outputs by the phase factors $\exp \left(-m \Delta \Phi_{d} / M\right), m=0,1, \cdots, M-1$. It is our experience that the oscillators' frequency offset can easily be tuned to $\Delta f / f<2 \times 10^{-10}$, which corresponds to a frequency difference of $0.4 \mathrm{~Hz}$ at $1900 \mathrm{MHz}$.
A note is to be made about the assumed stationarity of the radio channel during the measurements. In real mobile environments, complete channel stability is impossible to achieve, which is principally due to passing vehicles and movements of vegetation such as trees caused by wind. In our deterministic approach toward the characterization of the mobile radio channel we are interested in the influence of constant features of the built-up environment rather than in the effects of moving objects which cannot be modeled by any database. Wind effects can be largely avoided if measurements are conducted during days when there is little or no wind. As for moving vehicles, we believe that the statistical averaging of many measurements taken over several minutes of time will strongly reduce their interference, which typically lasts for several seconds. This averaging over many measurements ("snapshots") is inherent to subspace based angular superresolution methods such as UCA-MUSIC, as will be discussed in Section IV.

\section{Antennas}

Considerable attention has been paid to the choice of the antennas for our measurement setup. In the estimation of 2-D AoA's with a UCA, there exists a twofold ambiguity with respect to waves coming from the upper and the lower hemisphere, as is the case for any type of planar array. As we are primarily interested in multipath contributions impinging from above the array, we would like the receiving antenna to have lower sensitivity toward contributions with negative elevation angles. In addition, the applied angular superresolution algorithm assumes the array elements to have constant gain in the azimuth plane. Therefore, we used a $2-\mathrm{dBi}$ sleeve antenna with an omnidirectional radiation pattern in the azimuth plane, a vertical $3-\mathrm{dB}$ beamwidth of $60^{\circ}$ and maximum directivity for an elevation of $+20^{\circ}$. The antenna bandwidth extends from 1.7 to $2.0 \mathrm{GHz}$.

In outdoor radio channel measurements, the receiving antenna is often mounted on the roof of a vehicle. Although it is usually assumed that the vehicle roof will completely block all signals coming from below the horizontal plane [2], diffraction of multipath waves by the edges of the vehicle roof will in reality result in distortion of the azimuthal symmetry and the introduction of significant secondary lobes pointed toward the ground. To reduce the effect of the vehicle on the antenna radiation pattern and conserve its azimuthal symmetry, we have placed the antenna over a circular ground plane with diameter $D=5 \lambda$, where $\lambda$ denotes the wavelength. The measured azimuth and elevation patterns of this antenna configuration are shown in Fig. 3.

At the transmitter end we used a directive antenna to exploit the full dynamic range of our channel sounding system even in situations with high path loss. The antenna is a 7.1- $\mathrm{dBi}$ vertically polarized double-ridged pyramidal horn antenna with an azimuthal $3-\mathrm{dB}$ beamwidth of $53^{\circ}$, which is sufficient for a more or less uniform illumination of an area of microcellular dimensions close to the transmitter. The bandwidth of this antenna covers the frequency region from 1 to $18 \mathrm{GHz}$. 


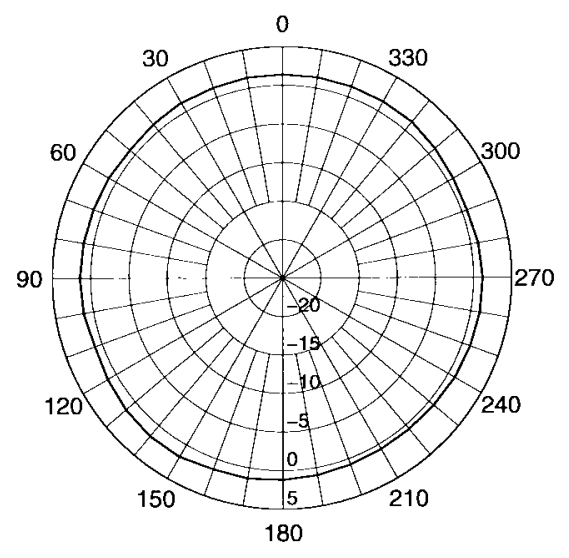

(a)

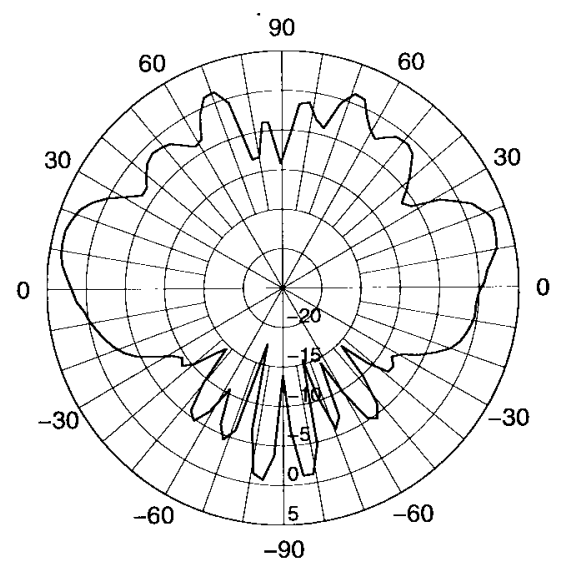

(b)

Fig. 3. Measured radiation patterns of the receiving antenna with ground plane $(D / \lambda=5)$. (a) Azimuth pattern $(H$-plane). (b) Elevation pattern $(E$-plane).

\section{DATA MODEL}

In the considered data model, the PN sounding signal $a(t)$ has a period $T$ and the bit duration is $T_{c}$. The electromagnetic field around the mobile unit is assumed to be composed of $N$ plane wave multipath contributions arriving from different angles. The mobile is equipped with a UCA of radius $r$ consisting of $M$ vertically polarized antenna elements with an omnidirectional azimuth pattern [as in Fig. 3(a)]. The correlation signal $y_{m}\left(\tau_{i}\right), i=0,1,2, \cdots$ at the $m$ th antenna element $(m=0,1, \cdots, M-1)$ can then be written as

$$
y_{m}\left(\tau_{i}\right)=\sum_{n=1}^{N} c_{n} g\left(\zeta_{n}\right) e^{j \zeta_{n} \cos \left(\varphi_{n}-\gamma_{m}\right)} x\left(\tau_{i}-T_{n}\right)+\eta_{m}\left(\tau_{i}\right)
$$

with $\tau_{i}=i T_{c}+\Delta \tau, \Delta \tau$ being a random delay offset with uniform probability density on the interval $0<\Delta \tau<T_{c}$. This delay offset is realized through the programmable delay unit in Fig. 1(b). The interval $\left(i T_{c},(i+1) T_{c}\right)$ will be referred to as the $i$ th delay interval or delay bin. In the above expression, $\gamma_{m}=2 \pi m / M$ is the azimuth of the $m$ th antenna element (view Fig. 2), $c_{n}$ and $T_{n}$ are the complex amplitude and the relative delay of the $n$th multipath wave, respectively, $\varphi_{n}$ is its azimuth angle, and $\zeta_{n}:=2 \pi r \cos \left(\vartheta_{n}\right) / \lambda$ represents the elevation dependence. Here, $\vartheta_{n}$ is the elevation of the $n$th wave. Further, $g(\zeta)$ is the elevation voltage pattern of the array elements [which can be obtained from Fig. 3(b)]

$$
x(\tau)=\frac{1}{T} \int_{0}^{T} a(t) a(t-\tau) d t
$$

is the autocorrelation function of the applied PN maximum length bit sequence and $\eta_{m}(\tau)$ is an additive noise signal. The autocorrelation function $x(\tau)$ is a triangular function with a spread of $\pm T_{c}$ around the peak value and with negligible sidelobes ( $54 \mathrm{~dB}$ below the peak value), as illustrated in Fig. 4. In (1), the time in which the radio waves travel across the array is assumed to be negligible compared with the bit period $T_{c}$ (narrow-band array assumption). Also, the effects of the limited bandwidth of the channel sounding system have been neglected. For later use and for notational convenience, we

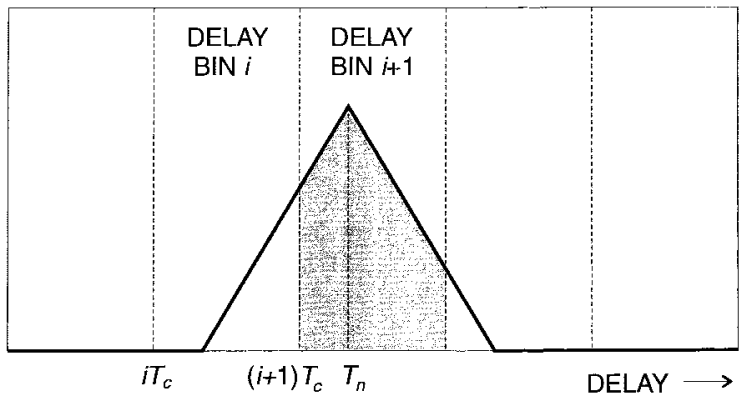

Fig. 4. Example of the correlation signal due to a multipath with delay $T_{n}$.

here define $\theta:=\zeta e^{j \varphi}$, which represents both azimuth and elevation.

Due to the limited width of the autocorrelation function $x(\tau)$ each received multipath wave contributes to at most three subsequent delay bins, and its contributions to the other delay bins are negligible (see Fig. 4). Omitting all $N-N^{(i)}$ negligible contributions to the $i$ th delay bin, (1) can be rewritten as

$$
\begin{aligned}
y_{m}\left(\tau_{i}\right)= & \sum_{n=1}^{N^{(i)}} c_{n}^{(i)} g\left(\zeta_{n}^{(i)}\right) e^{j \zeta_{n}^{(i)} \cos \left(\varphi_{n}^{(i)}-\gamma_{m}\right)} \\
& \cdot x\left(\tau_{i}-T_{n}^{(i)}\right)+\eta_{m}\left(\tau_{i}\right)
\end{aligned}
$$

where $c_{n}^{(i)}, T_{n}^{(i)}, \varphi_{n}^{(i)}$, and $\zeta_{n}^{(i)}$ are the re-indexed amplitudes, delays, azimuth angles, and elevation dependences, respectively, of the $N^{(i)} \leq N$ nonnegligible multipath signals contributing to the $i$ th delay interval.

In vector notation, the UCA response at the $i$ th delay instant is given by

$$
\begin{aligned}
\mathbf{y}\left(\tau_{i}\right) & =\left[y_{0}\left(\tau_{i}\right), y_{1}\left(\tau_{i}\right), \cdots, y_{M-1}\left(\tau_{i}\right)\right]^{T} \\
& =\sum_{n=1}^{N^{(i)}} \mathbf{u}\left(\theta_{n}^{(i)}\right) s_{n}^{(i)}\left(\tau_{i}\right)+\boldsymbol{\eta}\left(\tau_{i}\right)
\end{aligned}
$$

where $s_{n}^{(i)}\left(\tau_{i}\right)=c_{n}^{(i)} g\left(\zeta_{n}^{(i)}\right) x\left(\tau_{i}-T_{n}^{(i)}\right)$

$$
\mathbf{u}(\theta)=\left[e^{j \zeta \cos \left(\varphi-\gamma_{0}\right)}, e^{j \zeta \cos \left(\varphi-\gamma_{1}\right)}, \cdots, e^{j \zeta \cos \left(\varphi-\gamma_{M}-1\right)}\right]^{T}
$$


represents the array steering vector in the direction represented by $\theta$ and

$$
\boldsymbol{\eta}\left(\tau_{i}\right)=\left[\eta_{0}\left(\tau_{i}\right), \eta_{1}\left(\tau_{i}\right), \cdots, \eta_{M-1}\left(\tau_{i}\right)\right]^{T}
$$

contains the noise signals at the array elements. In the above expressions, the superscript $T$ denotes transpose. In matrix notation, (4) is rewritten as

$$
\mathbf{y}\left(\tau_{i}\right)=\mathbf{U}^{(i)} \mathbf{S}^{(i)}\left(\tau_{i}\right)+\boldsymbol{\eta}\left(\tau_{i}\right)
$$

where

$$
\begin{aligned}
\mathbf{U}^{(i)} & =\left[\mathbf{u}\left(\theta_{1}^{(i)}\right), \mathbf{u}\left(\theta_{2}^{(i)}\right), \cdots, \mathbf{u}\left(\theta_{N^{(i)}}^{(i)}\right)\right], \\
\mathbf{s}^{(i)}\left(\tau_{i}\right) & =\left[s_{1}^{(i)}\left(\tau_{i}\right), s_{2}^{(i)}\left(\tau_{i}\right), \cdots, s_{N^{(i)}}^{(i)}\left(\tau_{i}\right)\right]^{T} .
\end{aligned}
$$

The noise at each array element is assumed to be uncorrelated with the signals and the noise at the other elements and also to have zero mean and variance $\sigma^{2}$.

In conventional, nonadaptive beamforming [9], the array element output signals are phase-shifted and combined in such a way that they add up coherently for a given direction. Expressed in terms of a vector inner product, the response of the UCA with the main beam pointed in the direction represented by $\theta^{\prime}$ at delay instant $\tau_{i}$ then is

$$
\begin{aligned}
R\left(\theta^{\prime} ; \tau_{i}\right) & =\mathbf{u}^{\dagger}\left(\theta^{\prime}\right) \mathbf{y}\left(\tau_{i}\right) \\
& =M \sum_{n=1}^{N^{(i)}} D\left(\theta_{n}^{(i)} ; \theta^{\prime}\right) s_{n}^{(i)}\left(\tau_{i}\right)+\mathbf{u}^{\dagger}\left(\theta^{\prime}\right) \boldsymbol{\eta}\left(\tau_{i}\right)
\end{aligned}
$$

where $D\left(\theta ; \theta^{\prime}\right):=\mathbf{u}^{\dagger}\left(\theta^{\prime}\right) \mathbf{u}(\theta) / M$ represents the normalized array factor with the main lobe pointed in the direction associated with $\theta^{\prime}$. The superscript $\dagger$ denotes conjugate transpose. Using Graf's addition theorem for Bessel functions [10], it can be shown that

$$
D\left(\theta ; \theta^{\prime}\right)=J_{0}\left(\left|\theta-\theta^{\prime}\right|\right)
$$

provided that $M \gg \min \left(\zeta, \zeta^{\prime}\right)$. Here, $J_{h}(\cdot)$ denotes the Bessel function of the first kind of order $h$.

The half-power beamwidth (HPBW) of an antenna (array) is normally defined in a specified radiation pattern cut as the angle between the two directions in which the directivity is one-half the maximum value. If we express the angular separation between two directions $\left(\varphi_{1}, \zeta_{1}\right)$ and $\left(\varphi_{2}, \zeta_{2}\right)$ in the complex $\theta$-plane as the distance $\Delta \theta=\left|\theta_{1}-\theta_{2}\right|$ between the corresponding $\theta_{1}$ and $\theta_{2}$, it is seen from (10) that the array HPBW expressed in $\Delta \theta$ is constant over the entire field-ofview. It is further seen that conventional beamforming with UCA's results in high sidelobe levels in the array beam pattern due to the behavior of $J_{0}(\cdot)$. This latter property, together with our objective to obtain high-resolution AoA estimates even with a relatively small UCA radius, forms the rationale behind our choice for a superresolution algorithm for AoA estimation (UCA-MUSIC), which will be discussed in the next section. Angular superresolution techniques such as MUSIC are well known to offer a significantly better resolution performance than conventional beamformers of which the resolution is generally limited by the array HPBW.

\section{ANGULAR SUPERRESOLUTION}

\section{A. Beamspace Processing}

The elements of the vector $\mathbf{u}(\theta)$ may be thought of as samples of the signal

$$
u(\gamma, \theta)=e^{j \zeta \cos (\varphi-\gamma)}
$$

which is periodic in $\gamma$ with period $2 \pi$ and can, therefore, be represented by the Fourier series [10]

$$
\begin{aligned}
u(\gamma, \theta) & =\sum_{h=-\infty}^{\infty} j^{|h|} a_{h}(\theta) e^{-j h \gamma} \\
a_{h}(\theta) & =\frac{1}{2 \pi j|h|} \int_{0}^{2 \pi} u(\gamma, \theta) e^{j h \gamma} d \gamma=J_{|h|}(\zeta) e^{j h \varphi} .
\end{aligned}
$$

Although the spectral width of the signal $u(\gamma, \theta)$ is infinite in theory, the magnitudes $J_{|h|}(\zeta)$ of the Fourier coefficients decrease fast for $|h|>\zeta$ and are, in fact, bounded by [6]

$$
\left|J_{|h|}(\zeta)\right| \leq\left(\frac{\zeta e}{2|h|}\right)^{|h|} \leq\left(\frac{\pi r e}{\lambda|h|}\right)^{|h|}, \quad h \neq 0 .
$$

For sufficiently large $|h|$, the coefficients $a_{h}(\theta)$ for which $|h|>\pi r e / \lambda$ become negligibly small. Aliasing can hence be avoided by choosing the number of array elements (the spatial sample frequency) $M>2 \pi \mathrm{re} / \lambda$, and we are only interested in the nonnegligible coefficients $a_{h}(\theta),-H \leq h \leq H$, with $H=\lfloor\pi$ re $/ \lambda\rfloor$, where $\lfloor\cdot\rfloor$ is the largest integer smaller than the argument. Note that in the present case $(M=106, r=0.30$ $\mathrm{m}, \lambda=0.16 \mathrm{~m})$, the above condition is well satisfied.

A unit-norm vector of coefficients

$$
\mathbf{a}(\theta)=\left[a_{-H}(\theta), \cdots, a_{0}(\theta), \cdots, a_{H}(\theta)\right]^{T}
$$

can be obtained directly from $\mathbf{u}(\theta)$ by the transformation

$$
\mathbf{a}(\theta)=\frac{1}{\sqrt{M}} \mathbf{C V u}(\theta)
$$

where

$$
\begin{aligned}
\mathbf{C} & =\operatorname{diag}\left\{j^{-H}, \cdots, j^{0}, \cdots, j^{-H}\right\} \\
\mathbf{V} & =\frac{1}{\sqrt{M}}\left[\mathbf{v}_{0}, \mathbf{v}_{1}, \cdots, \mathbf{v}_{M-1}\right] \\
\mathbf{v}_{m} & =\left[\omega^{-m H}, \cdots, \omega^{0}, \cdots, \omega^{m H}\right]^{T}
\end{aligned}
$$

$\omega=\exp (j 2 \pi / M)$, which is based on the $(2 H+1) \times M$ submatrix of the spatial discrete Fourier transform [11], [12]. Multiplication of (7) by the matrix CV yields

$$
\mathbf{z}\left(\tau_{i}\right)=\mathbf{C V y}\left(\tau_{i}\right)=\mathbf{A}^{(i)} \mathbf{s}^{(i)}\left(\tau_{i}\right)+\mathbf{n}\left(\tau_{i}\right)
$$

where

$$
\begin{aligned}
\mathbf{A}^{(i)} & =\sqrt{M}\left[\mathbf{a}\left(\theta_{1}^{(i)}\right), \mathbf{a}\left(\theta_{2}^{(i)}\right), \cdots, \mathbf{a}\left(\theta_{N^{(i)}}^{(i)}\right)\right] \\
\mathbf{n}\left(\tau_{i}\right) & =\mathbf{C V} \eta\left(\tau_{i}\right) .
\end{aligned}
$$

Processing of the array measurement data in the spatial frequency domain is referred to as phase-mode excitation-based beamspace processing [11] and has important advantages over element space processing such as reduced sensitivity to noise and modeling errors [9] and the possibility to employ forward/backward averaging [11], as will be discussed in Section IV-C. 


\section{B. UCA-MUSIC}

The UCA-MUSIC algorithm uses the eigenstructure of the beamspace array output covariance matrix as a basis for AoA estimation. In the present case, covariance matrices are estimated for all time delay intervals $\left(i T_{c},(i+1) T_{c}\right)$, $i=0,1, \cdots$, and for each interval UCA-MUSIC provides AoA estimates of the $N^{(i)}$ multipath signals that contribute significantly to the measured channel response. The covariance matrix corresponding to the $i$ th delay bin can be expressed as

$$
\mathbf{R}^{(i)}=\mathrm{E}\left\{\mathbf{z}\left(\tau_{i}\right) \mathbf{z}^{\dagger}\left(\tau_{i}\right)\right\}=\mathbf{A}^{(i)} \mathbf{S}^{(i)} \mathbf{A}^{(i) \dagger}+\sigma^{2} \mathbf{I}
$$

where $\mathbf{S}^{(i)}=\mathrm{E}\left\{\mathbf{s}^{(i)}\left(\tau_{i}\right) \mathbf{s}^{(i) \dagger}\left(\tau_{i}\right)\right\}$ is the signal covariance matrix. In the last expression, use has been made of the fact that $\mathbf{C V} \mathbf{V}^{\dagger} \mathbf{C}^{\dagger}=\mathbf{I}$.

Let $\lambda_{1}^{(i)} \geq \lambda_{2}^{(i)} \geq \cdots \geq \lambda_{L}^{(i)}$ denote the $L=2 H+1$ eigenvalues of $\mathbf{R}^{(i)}$, and $\mathbf{e}_{1}^{(\bar{i})}, \mathbf{e}_{2}^{(i)}, \cdots, \mathbf{e}_{L}^{(i)}$ the corresponding normalized eigenvectors. Provided that $\mathbf{S}^{(i)}$ is nonsingular, $\mathbf{A}^{(i)} \mathbf{S}^{(i)} \mathbf{A}^{(i) \dagger}$ is of rank $N^{(i)}$, and it follows that $\lambda_{l}^{(i)}>\sigma^{2}$, $l=1, \cdots, N^{(i)}$ and $\lambda_{l}^{(i)}=\sigma^{2}, l=N^{(i)}+1, \cdots, L$, as long as $N^{(i)}<L$. Furthermore, $\mathbf{e}_{l}^{(i) \dagger} \mathbf{a}\left(\theta_{n}^{(i)}\right)=0, l=N^{(i)}+1, \cdots, L$; $n=1, \cdots, N^{(i)}$, i.e., the eigenvectors associated with the $L-N^{(i)}$ smallest eigenvalues (spanning the noise subspace) are orthogonal to the space spanned by the $N^{(i)}$ beamspace steering vectors (the signal subspace) [13]. MUSIC-based estimation algorithms exploit this orthogonality property to form a null spectrum, which is, in our case, given by

$$
Q^{(i)}(\theta)=\sum_{l=N^{(i)}+1}^{L}\left|\mathbf{e}_{l}^{(i) \dagger} \mathbf{a}(\theta)\right|^{2}=1-\sum_{l=1}^{N^{(i)}}\left|\mathbf{e}_{l}^{(i) \dagger} \mathbf{a}(\theta)\right|^{2} .
$$

A 2-D search for the nulls of $Q^{(i)}(\theta)$ over the full field-ofview yields unbiased and zero-variance estimates of the $N^{(i)}$ actual AoA's, regardless of signal-to-noise ratio (SNR) and angular spacing between the sources. The corresponding signal strengths can be obtained via the least-squares solution to (19).

\section{Forward/Backward Averaging}

Eigenstructure based superresolution algorithms such as UCA-MUSIC fail when the source covariance matrix $\mathbf{S}^{(i)}$ is singular, which occurs when two or more signals are perfectly correlated or coherent. In subspace-based AoA estimation based on a single-data snapshot [2], all signals are coherent unless a remedy called spatial smoothing is applied, which is well known to decrease the effective array aperture and, hence, the angular estimation accuracy and resolution capability [9]. In our case, complete signal coherence occurs only when two or more multipaths have exactly the same propagation delay. This is an important benefit of the averaging over a number of snapshots with different random delay offsets. Still, in practice, UCA-MUSIC will be less accurate when two or more signals are strongly correlated, i.e., when the difference between the delays of two multipaths is very small compared to $T_{c}$. Therefore, in order to further reduce the correlation between the signals, we apply forward/backward (FB) averaging of the covariance matrix [11], [14], a technique which hinges on the centro-Hermitian property of the beamspace array steering vector $\mathbf{a}(\theta){ }^{1}$

The FB averaged covariance matrix is

$$
\tilde{\mathbf{R}}^{(i)}=\frac{1}{2}\left(\mathbf{R}^{(i)}+\mathbf{J R}^{(i) *} \mathbf{J}\right)=\mathbf{A}^{(i)} \tilde{\mathbf{S}}^{(i)} \mathbf{A}^{(i) \dagger}+\sigma^{2} \mathbf{I}
$$

where $\tilde{\mathbf{S}}^{(i)}=\left(\mathbf{S}^{(i)}+\mathbf{S}^{(i) *}\right) / 2$ is the FB averaged signal covariance matrix. The superscript $*$ denotes complex conjugate. The eigenvalues of $\tilde{\mathbf{R}}^{(i)}$ are denoted by $\tilde{\lambda}_{1}^{(i)} \geq \tilde{\lambda}_{2}^{(i)} \geq$ $\cdots \geq \tilde{\lambda}_{L}^{(i)}$ and the corresponding normalized eigenvectors by $\tilde{\mathbf{e}}_{1}^{(i)}, \tilde{\mathbf{e}}_{2}^{(i)}, \cdots, \tilde{\mathbf{e}}_{L}^{(i)}$. Result of the FB averaging preprocessing is that the magnitudes of the off-diagonal terms of $\mathbf{S}^{(i)}$, representing the cross-correlations between all pairs of signals $s_{m}^{(i)}\left(\tau_{i}\right), s_{n}^{(i)}\left(\tau_{i}\right), m \neq n$, are effectively multiplied by a factor $\cos \left(\phi_{m n}\right)$, where $\phi_{m n}$ represents the phase difference between the $m$ th and the $n$th signal. Thus, FB averaging improves the condition of the array output covariance matrix and consequently the performance of the UCA-MUSIC algorithm.

\section{Effects of Finite Data}

In our measurements, the covariance matrix $\tilde{\mathbf{R}}^{(i)}$ is estimated from a finite number of beamspace data snapshots $\mathbf{z}\left(\tau_{i ; k}\right)$, with $\tau_{i ; k}=\tau_{i}+\Delta \tau_{k}, k=1,2, \cdots, K$, where $\Delta \tau_{k}$ is a realization of the random delay offset $\Delta \tau$. The resulting estimated covariance matrix is

$$
\hat{\mathbf{R}}^{(i)}=\frac{1}{2 K} \sum_{k=1}^{K}\left(\mathbf{z}\left(\tau_{i ; k}\right) \mathbf{z}^{\dagger}\left(\tau_{i ; k}\right)+\mathbf{J}^{*}\left(\tau_{i ; k}\right) \mathbf{z}^{T}\left(\tau_{i ; k}\right) \mathbf{J}\right)
$$

with eigenvalues $\hat{\lambda}_{1}^{(i)} \geq \hat{\lambda}_{2}^{(i)} \geq \cdots \geq \hat{\lambda}_{L}^{(i)}$ and corresponding normalized eigenvectors $\hat{\mathbf{e}}_{1}^{(i)}, \hat{\mathbf{e}}_{2}^{(i)}, \cdots, \hat{\mathbf{e}}_{L}^{(i)}$. Now, the estimated null spectrum is written as

$$
\hat{Q}^{(i)}(\theta)=\sum_{l=N^{(i)}+1}^{L}\left|\hat{\mathbf{e}}_{l}^{(i) \dagger} \mathbf{a}(\theta)\right|^{2}=1-\sum_{l=1}^{N^{(i)}}\left|\hat{\mathbf{e}}_{l}^{(i) \dagger} \mathbf{a}(\theta)\right|^{2}
$$

and the AoA estimates are obtained by searching for the $N^{(i)}$ deepest nulls. In general, the estimated matrix $\hat{\mathbf{R}}^{(i)}$ differs from the true FB averaged covariance matrix $\tilde{\mathbf{R}}^{(i)}$, which causes a perturbation of the estimated null spectrum from the true null spectrum. This in turn causes errors in the AoA estimates and limits the capability to discriminate between closely spaced sources (resolution threshold).

Another consequence of the estimation of $\tilde{\mathbf{R}}^{(i)}$ from finite data is that the eigenvalues become all different with probability one so that it is no longer straightforward to estimate the dimensionality of the noise subspace. However, an objective estimate of the number of multipath signals contributing to the considered time delay bin can be obtained by applying a modified version of Akaike's information criterion [14], which operates on the FB averaged covariance matrix (FB-AIC).

\footnotetext{
${ }^{1} \mathrm{~A}$ complex vector $\mathbf{z}$ is defined to be centro-Hermitian if it satisfies $\mathbf{J} \mathbf{z}=\mathbf{z}^{*}$, where $\mathbf{J}$ is the reverse permutation matrix [11]
} 


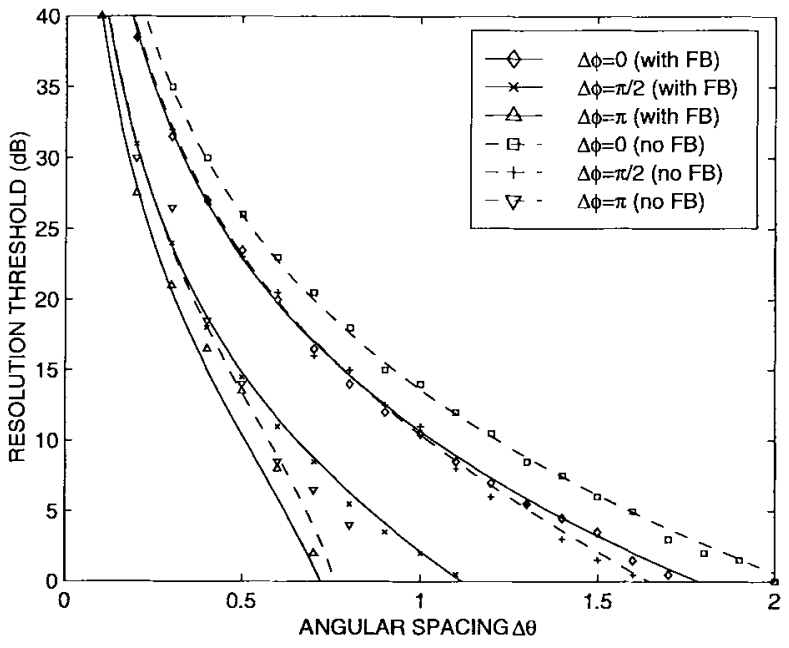

(a)

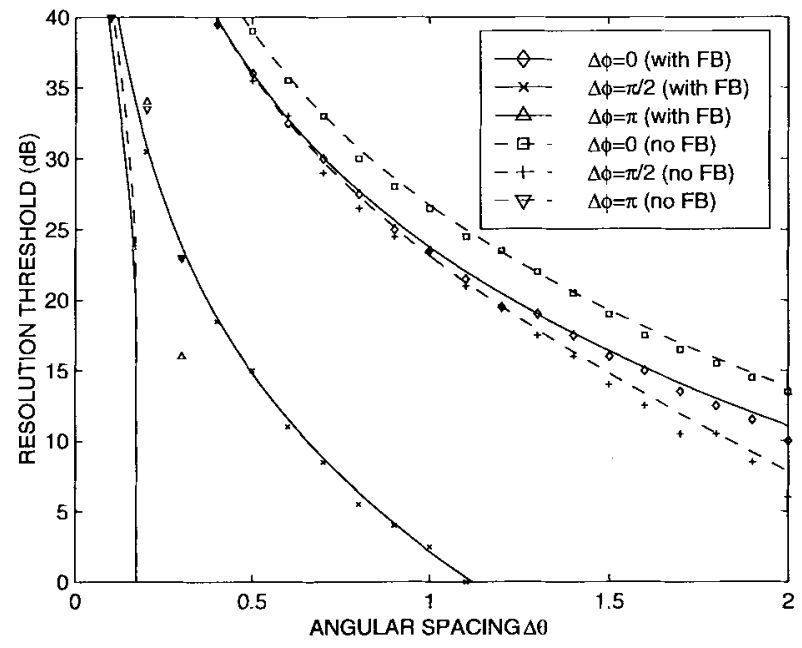

(b)

Fig. 5. Theoretical and simulated resolution thresholds for UCA-MUSIC with and without FB averaging for propagation delay differences: (a) $\Delta T=0.5 T_{c}$ and (b) $\Delta T=0.1 T_{c} . M=106, r=0.30 \mathrm{~m}, \lambda=0.158 \mathrm{~m}, K=20$ snaphots. Simulated thresholds are based on 50 independent trials.

\section{Simulation Results}

Computer simulations of the performance of the UCAMUSIC algorithm and the effect of FB averaging preprocessing are presented in this section. First, we show simulations of the resolution threshold [15], which is the SNR below which two closely spaced, equipowered multipath waves can no longer be "resolved," i.e., when the null spectrum at either of the two true AoA's $\theta_{1,2}$ is greater than at the angle $\theta_{m}=\left(\theta_{1}+\theta_{2}\right) / 2$ between the two true AoA's with more than $50 \%$ probability. Here, the SNR associated with each of the multipath signals and the $i$ th delay bin is defined as

$$
\mathrm{SNR}_{1,2}^{(i)}=\frac{1}{\sigma^{2} T_{c}} \int_{i T_{c}}^{(i+1) T_{c}}\left|s_{1,2}(\tau)\right|^{2} d \tau
$$

with $\sigma^{2}, T_{c}$, and $s_{1,2}(\tau)$, as defined previously.

Fig. 5 shows simulated and theoretical resolution thresholds for two multipaths of equal strength, as a function of the angular spacing $\Delta \theta=\left|\theta_{1}-\theta_{2}\right|$ for different delay differences $\Delta T$. In the simulation, the considered delay interval was the zeroth bin $\left(0, T_{c}\right)$, the multipaths' AoA's were chosen equal to $\theta_{1,2}= \pm \Delta \theta / 2, \Delta \theta$ being a real number and their respective delay times were $T_{1,2}=\left(T_{c} \pm \Delta T\right) / 2, \Delta T=0.1 T_{c}, 0.5 T_{c}$. The number of snapshots was taken to be $K=20$ and all other parameters were chosen the same as for the experimental arrangement described in Section II $(M=106, r=0.30 \mathrm{~cm}$, $\lambda=0.158 \mathrm{~m}$ ). Each of the simulated threshold values in Fig. 5 was based on 50 independent trials. The solid and dashed lines represent theoretical resolution thresholds (with and without FB averaging, respectively) based on the general expressions derived in [15].

The theoretical and simulated results presented in Fig. 5 show reasonable to good agreement. As expected, the resolution capability of UCA-MUSIC is seen to decrease with decreasing delay difference (and, hence, increasing signal correlation). With FB averaging however, this performance degradation is only limited, except when the phase difference $\phi$ approaches zero or $\pi$ and the decorrelation effectiveness is strongly reduced. The small improvements due to FB averaging for $\phi=0$ and $\phi=\pi$ that can still be seen in Fig. 5 are the result of the fact that this scheme effectively doubles the number of snapshots. It is remarked that, since the array's directional properties are uniform in the complex $\theta$-plane, the above results are representative of the resolution capability over the entire field-of-view.

Second, simulation results are presented which illustrate how poor estimates $\hat{N}$ of the number of paths $N$ impact the UCA-MUSIC null spectra and the AoA estimation. To this end, a scenario with five incident multipath waves was simulated, the properties of which are given in Table I. Fig. 6(a)-(c) show the null spectrum for $\hat{N}=4,5$, and 8 , respectively. The corresponding estimated AoA's and SNR's are given in Table I.

From Fig. 6 it is observed that a modest under or overestimation of $N$ does not lead to dramatic changes of the null spectrum. Increasing underestimation of $N$ generally leads to shallower local minima and ultimately to the vanishing of the local minima corresponding with the weakest multipath waves. On the other hand, a modest overestimation of $N$ introduces spurious local minima, which, however, hardly alter the positions of the deeper nulls corresponding with the true AoA's. Table I shows that the estimated signal strengths of the falsely detected waves are all around or below the noise level and, therefore, insignificant in comparison with the dominant multipath signals, which are of primary interest in our application. From these observations, it is concluded that it is better to tolerate a small overestimation of $N$ than to underestimate the number of incident waves, which would lead to undetected multipath contributions. Of the available objective criteria for detecting the number of sources from an FB averaged covariance matrix, FB-AIC, and FB-MDL [14]; the former scheme provides slightly higher estimates of $N$ for a small number of snapshots. Furthermore, for the scenario 
TABLE I

Theoretical and Estimated Multipath Parameters

\begin{tabular}{|c|c|c|c|c|c|c|c|c|c|c|c|c|}
\hline \multirow{2}{*}{$\mathrm{n}$} & \multicolumn{3}{|c|}{ theoretical } & \multicolumn{3}{|c|}{$\hat{N}=4$} & \multicolumn{3}{|c|}{$\hat{N}=5$} & \multicolumn{3}{|c|}{$\hat{N}=8$} \\
\hline & $\begin{array}{c}\varphi \\
(\operatorname{deg})\end{array}$ & $\zeta(-)$ & $\begin{array}{l}\mathrm{SNR} \\
\text { (dB) }\end{array}$ & $\begin{array}{c}\varphi \\
(\mathrm{deg})\end{array}$ & $\zeta(-)$ & $\begin{array}{l}\text { SNR } \\
\text { (dB) }\end{array}$ & $\begin{array}{c}\varphi \\
(\operatorname{deg})\end{array}$ & $\zeta(-)$ & $\begin{array}{l}\text { SNR } \\
\text { (dB) }\end{array}$ & $\begin{array}{c}\varphi \\
(\operatorname{deg})\end{array}$ & $\zeta(-)$ & $\begin{array}{l}\text { SNR } \\
\text { (dB) }\end{array}$ \\
\hline 1 & 0.0 & 5.00 & 30.0 & -0.3 & 4.99 & 30.5 & -0.3 & 4.99 & 29.6 & -0.1 & 4.98 & 30.7 \\
\hline 2 & +72.0 & 5.00 & 25.0 & +71.1 & 5.02 & 25.5 & +71.2 & 5.01 & 24.6 & +71.4 & 4.94 & 25.3 \\
\hline 3 & +144.0 & 5.00 & 20.0 & +143.0 & 5.24 & 20.9 & +142.6 & 5.16 & 19.6 & +143.6 & 5.08 & 20.0 \\
\hline 4 & -144.0 & 5.00 & 15.0 & -144.2 & 5.28 & 15.7 & -144.1 & 5.21 & 14.8 & -142.7 & 5.03 & 15.1 \\
\hline 5 & -72.0 & 5.00 & 10.0 & - & - & - & -68.8 & 5.17 & 10.1 & -71.2 & 5.40 & 11.4 \\
\hline 6 & - & - & - & - & - & - & - & - & - & +180.0 & 6.59 & -2.9 \\
\hline 7 & - & - & - & - & - & - & - & - & - & -35.0 & 6.29 & -5.3 \\
\hline 8 & - & - & - & - & - & - & - & - & - & +11.3 & 11.26 & -7.4 \\
\hline
\end{tabular}

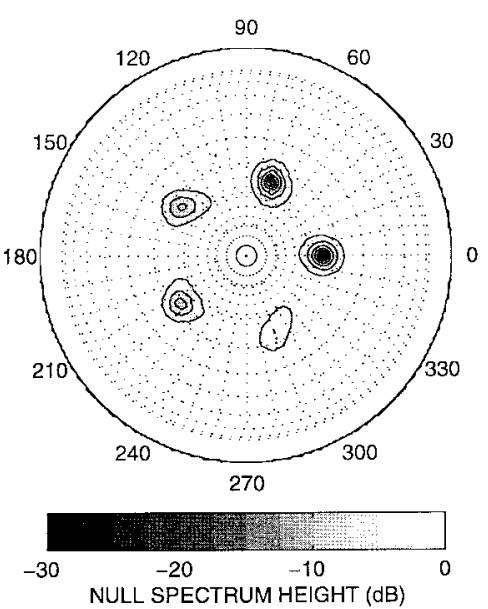

(a)

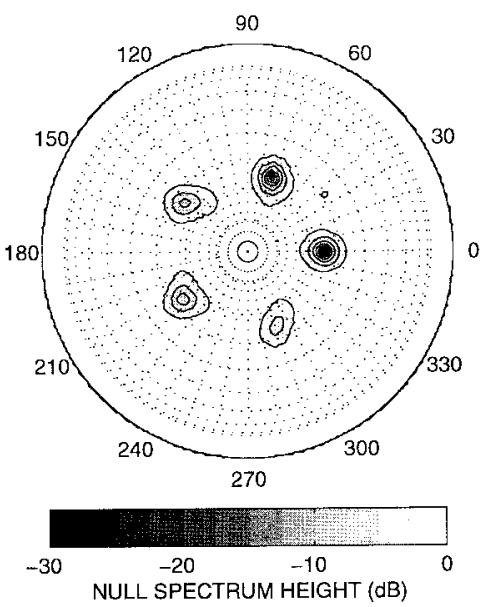

(b)

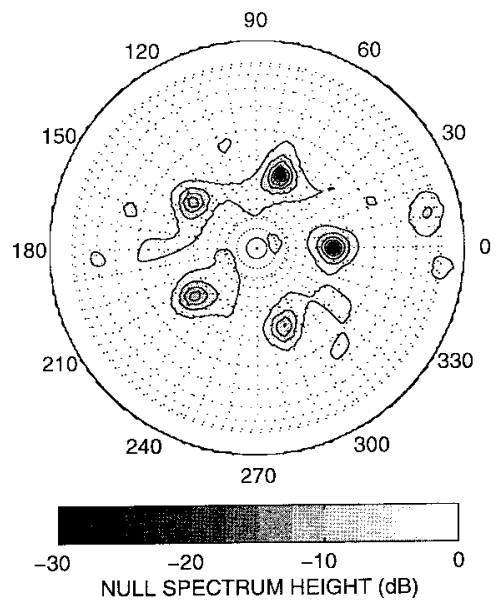

(c)

Fig. 6. UCA-MUSIC null spectra $\hat{Q}(\theta)$ for the simulated scenario. (a) $\hat{N}=4$. (b) $\hat{N}=5$. (c) $\hat{N}=8$. Dotted lines are drawn every $10^{\circ}$ azimuth and elevation (outer perimeter corresponds with $\vartheta=0^{\circ}$; center dot corresponds with $\vartheta= \pm 90^{\circ}$ ).

considered in [15], this scheme was shown to offer the highest probability of correctly detecting the number of waves for small values of $K$.

\section{EXPERIMENTAL RESULTS}

In this section, we will consider the results of two measurements carried out in a residential area in Leidschendam, The Netherlands, which is characterized by $5-15-\mathrm{m}$-high buildings, scattered vegetation, and low traffic density (view Fig. 7). During the measurements, the transmitting BS antenna (46 $\mathrm{m}$ above ground level) was pointed southeast. The number of snapshots in each measurement was $K=20$. Figs. 8 and 9 show the temporal and angular multipath distributions measured at the two mobile station (MS) locations together with $360^{\circ}$ panorama photographs taken from the receiver perspective, which serve as an accurate angular reference. In these figures, the marker size indicates the amplitude of each resolved multipath wave relative to the total power (which is obtained by integration of the measured power delay profile), elevation angles are represented through $\zeta=2 \pi r \cos (\vartheta) / \lambda$ and the BS antenna position is indicated by a dashed vertical line.

In the first measurement, the MS location (marked as "1" in Fig. 7) was chosen such that the direct wave was obstructed by a tree (T1 in Fig. 8). Scattering and absorption of the wave incident on the tree's canopy result in an attenuation of $13 \mathrm{~dB}$ over the free-space loss (although this is not shown here), and a considerable angular spread. The second peak in the measured power delay profile is due to the energy scattered from vegetation surrounding the MS $\left(-160<\varphi<0^{\circ}\right)$, which was directly illuminated by the BS antenna. The relatively weak contribution with delay $\tau \approx 1.5 \mu \mathrm{s}$ is the result of 


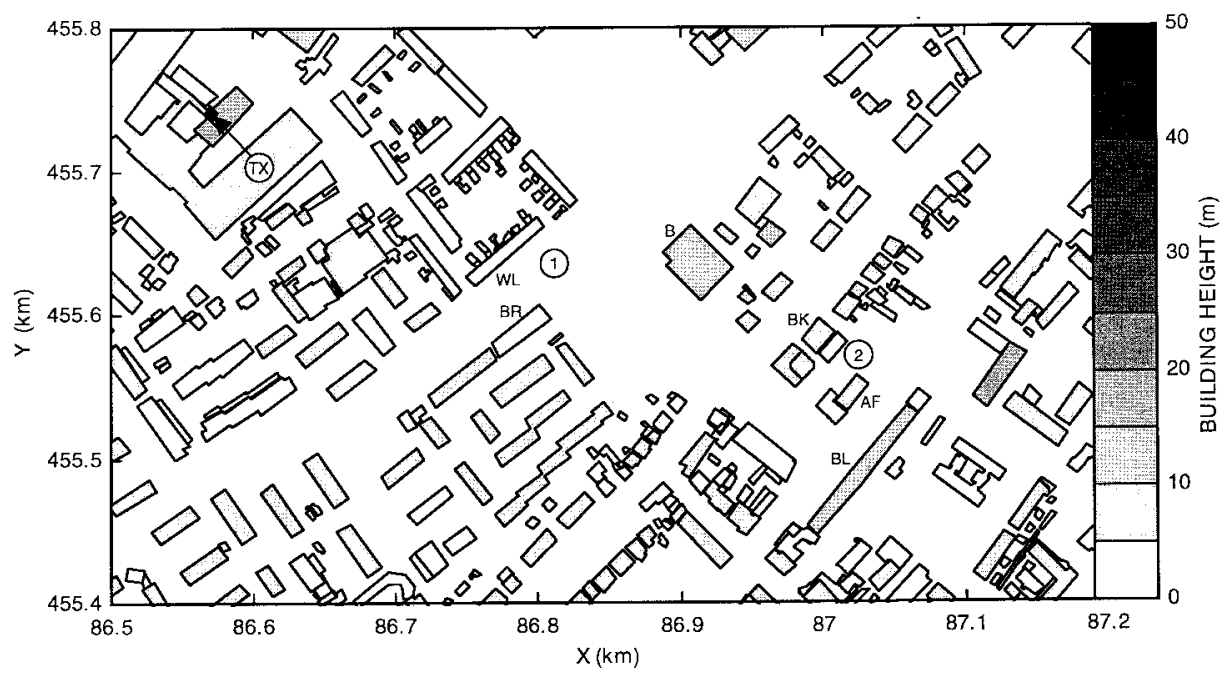

Fig. 7. Map of the measurement site. Gray levels indicate the building height in meters.

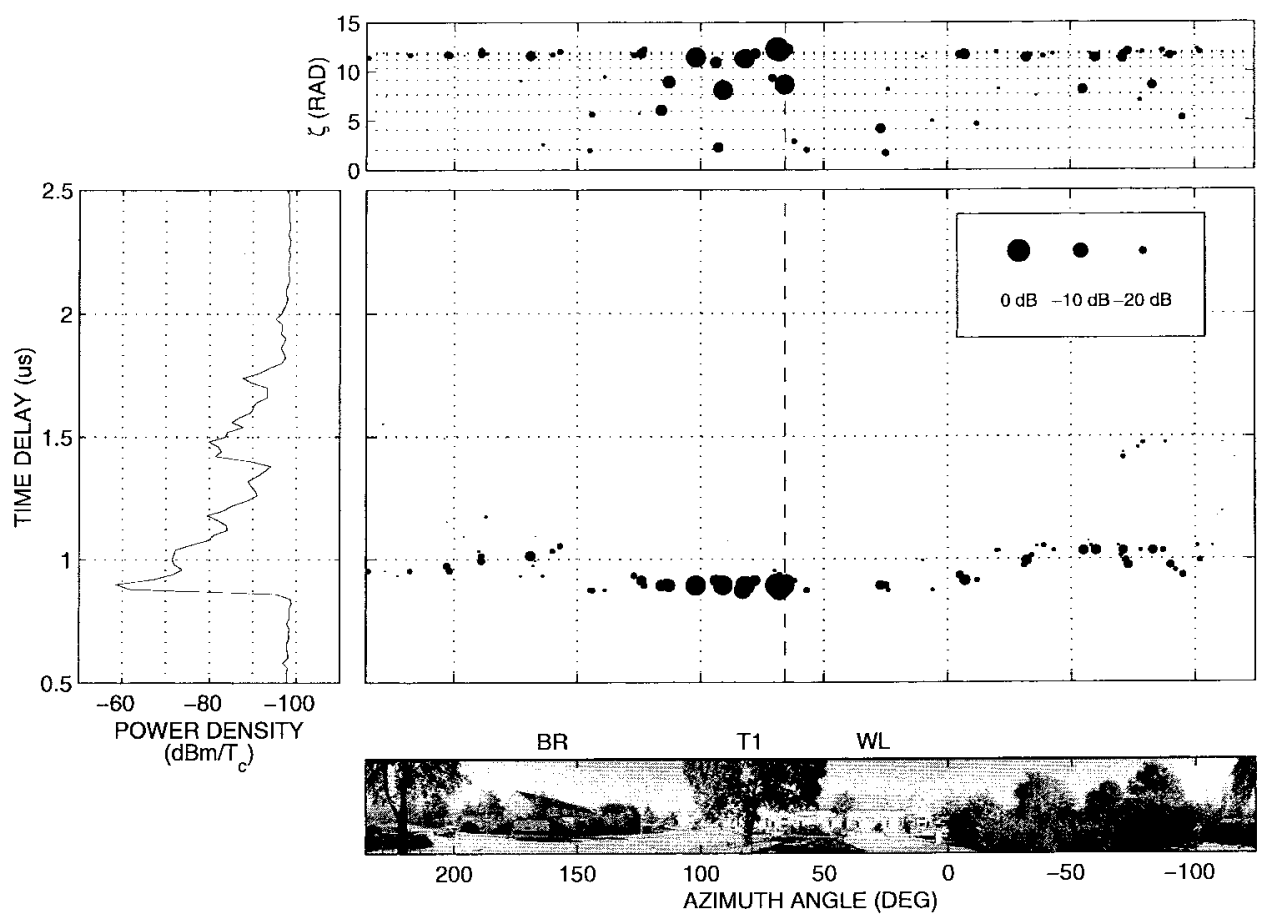

Fig. 8. Temporal and angular multipath distribution for MS location 1. Marker size indicates multipath wave amplitude relative to total power (down to $-30 \mathrm{~dB}$ ). Elevation angles are represented through $\zeta=2 \pi r \cos (\vartheta) / \lambda$. BS antenna position is indicated by dashed vertical line.

reflection and diffraction from the building $\mathrm{B}$ which is behind the vegetation.

In the second measurement, the MS was situated at a car park behind a 6-m-high building (location "2" in Fig. 7). Again, the first multipath contributions have a considerable angular spread, which is probably due to the row of trees between the transmitting antenna and the building BK. The diffraction contribution over the rooftop of BK is observed approximately at the expected azimuth angle of $70^{\circ}$. The wave with $\varphi=213^{\circ}$ can be identified (on the basis of both AoA and propagation delay) as a reflection from building BL. In this particular case, this reflected wave is the dominant contribution, due to the attenuation of the direct wave by the trees behind BK.

Another experiment conducted with the same method in an urban microcell environment with a low BS antenna has been presented in [16]. The results of this measurement have shown that transmission through buildings can be the dominant propagation mechanism in urban microcells.

\section{CONCLUSIONS}

In this paper, we have proposed a method to obtain highresolution AoA estimates of multipath waves using a wideband radio channel sounder and a synthetic uniform circular 


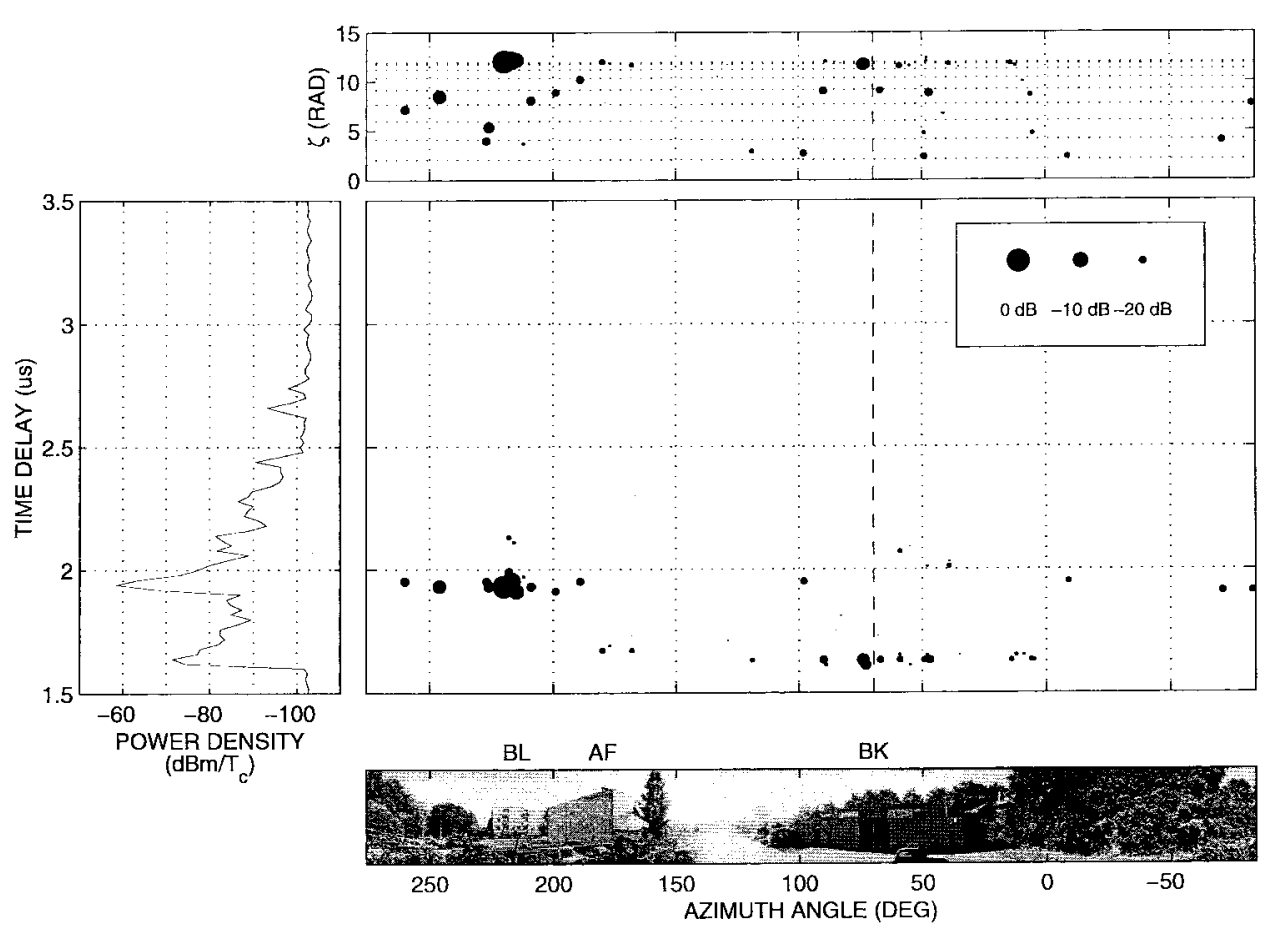

Fig. 9. Temporal and angular multipath distribution for MS location 2. Marker size indicates multipath wave amplitude relative to total power (down to $-30 \mathrm{~dB}$ ). Elevation angles are represented through $\zeta=2 \pi r \cos (\vartheta) / \lambda$. BS antenna position is indicated by dashed vertical line.

array. The UCA-MUSIC algorithm and forward/backward averaging were demonstrated to be suitable algorithms for the high-resolution 2-D (azimuth and elevation) AoA analysis of complex impulse response measurements obtained with this array configuration. We did not experience any difficulties with the algorithm with respect to its robustness to noise, as has been suggested in an earlier paper [6]. The problem of the $a$ priori estimation of the number of incident signals based on a finite set of measurement data was solved by applying a modified version of Akaike's information criterion (FB-AIC) [14].

It was demonstrated that the combination of high-resolution AoA estimates and time delay information enables the identification of the dominant rays in an actual mobile radio channel. The determination of the elevation angles of the impinging multipath waves permits to obtain additional information about the nature of the involved propagation mechanisms, although the estimation accuracy of elevation angles is relatively poor for waves arriving approximately from the horizontal plane. The resulting qualitative and quantitative insights into the propagation mechanisms involved in UHF radio propagation in built-up environments are important for the development of improved planning tools for microcellular mobile radio networks. Measurement results obtained with the proposed measurement technique have identified phenomena such as absorption and scattering by vegetation and transmission through buildings as important propagation mechanisms in mobile radio environments.

\section{ACKNOWLEDGMENT}

The authors would like to thank Dr. A. Mawira and J. Schmidt, KPN Research, Leidschendam, The Netherlands, for their valuable suggestions in the preparation of the experiments, and P. Rijsdijk, also with KPN Research, for his assistance during the measurement campaign. They would also like to thank K. van Staalduinen and J. van Bruggen, Ericsson Mobile Business Networks, Enschede, The Netherlands, for making available their channel sounding equipment. The authors are grateful to the anonymous reviewers for their comments and suggestions, which helped to improve this paper.

\section{REFERENCES}

[1] F. Ikegami and S. Yoshida, "Analysis of multipath propagation structure in urban mobile radio environments," IEEE Trans. Antennas Propagat., vol. AP-28, pp. 531-537, July 1980.

[2] J. Fuhl, J.-P. Rossi, and E. Bonek, "High-resolution 3-D direction-ofarrival determination for urban mobile radio," IEEE Trans. Antennas Propagat., vol. 45, pp. 672-682, Apr. 1997.

[3] J. G. O. Moss, A. M. Street, and D. J. Edwards, "Wideband radio imaging technique for multipath environments," Electron Lett., vol. 33, no. 11, pp. 941-942, 1997.

[4] Y. L. C. de Jong and M. H. A. J. Herben, "Accurate identification of scatterers for improved microcell propagation modeling," in Proc. 8th IEEE Int. Symp. Personal, Indoor, Mobile Radio Commun. (PIMRC'97), Helsinki, Finland, Sept. 1997, pp. 645-649.

[5] K. I. Pedersen, B. H. Fleury, and P. E. Mogensen, "High resolution of electromagnetic waves in time-varying radio channels," in Proc. 8th IEEE Int. Symp. Personal, Indoor, Mobile Radio Commun. (PIMRC'97), Helsinki, Finland, Sept. 1997, pp. 650-654.

[6] J.-P. Rossi, J.-P. Barbot, and A. J. Levy, "Theory and measurement of the angle of arrival and time delay of UHF radiowaves using a ring array," IEEE Trans. Antennas Propagat., vol. 45, pp. 876-884, May 1997.

[7] T. Manabe and H. Takai, "Superresolution of multipath delay profiles measured by PN correlation method," IEEE Trans. Antennas Propagat., vol. 40, pp. 500-509, May 1992.

[8] D. C. Cox, "Delay Doppler characteristics of multipath propagation at $910 \mathrm{MHz}$ in a suburban mobile radio environment," IEEE Trans. Antennas Propagat., vol. AP-20, pp. 625-635, Sept. 1972. 
[9] L. C. Godara, "Appliations of antenna arrays to mobile communications-Part II: Beamforming and direction-of-arrival considerations," Proc. IEEE, vol. 85, pp. 1195-1245, Aug. 1997.

[10] M. Abramowitz and I. A. Stegun, Handbook of Mathematical Functions. New York: Dover, 1965.

[11] C. P. Mathews and M. D. Zoltowski, "Eigenstructure techniques for 2D angle estimation with uniform circular arrays," IEEE Trans. Signal Processing, vol. 42, pp. 2395-2407, Sept. 1994.

[12] M. Wax and J. Sheinvald, "Direction finding of coherent signals via spatial smoothing for uniform circular arrays," IEEE Trans. Antennas Propagat., vol. 42, pp. 613-620, May 1994.

[13] R. O. Schmidt, "Multiple emitter location and signal parameter estimation," IEEE Trans. Antennas Propagat., vol. AP-34, pp. 276-280, Mar. 1986.

[14] G. Xu, R. H. Roy, and T. Kailath, "Detection of number of sources via exploitation of centro-symmetry property," IEEE Trans. Signal Processing, vol. 42, pp. 102-112, Jan. 1994.

[15] L. Chang and C.-C. Yeh, "Resolution threshold for coherent sources using smoothed eigenstructure methods," Proc. Inst. Elect. Eng., vol 138, pt. F, no. 5, pp. 470-478, Oct. 1991.

[16] Y. L. C. de Jong, M. H. A. J. Herben, J.-F. Wagen, and A. Mawira, "Transmission of UHF radiowaves through buildings in urban microcell environments," Electron. Lett., vol. 35, no. 9, pp. 743-745, 1999.

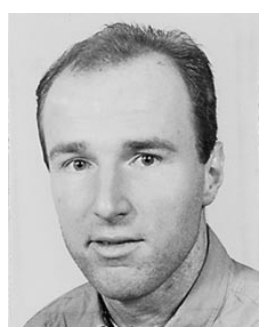

Yvo L. C. de Jong (S'96) was born in Vlaardingen, The Netherlands, in 1972. He received the M.Sc. degree (cum laude) in electrical engineering from Eindhoven University of Technology (EUT), Eindhoven, The Netherlands, in 1996. He is currently working toward the Ph.D. degree at the same institute.

In the framework of his Ph.D. project, he was with the Signal Transport Systems Group, KPN Research, The Netherlands, from January 1997 to May 1998, and with Swisscom Corporate Information and Technology, Switzerland, from June to October 1998. His research includes deterministic propagation modeling for mobile communications.

Mr. de Jong is a member of the Royal Institute of Engineers (Klvl) in The Netherlands and the Netherlands Electronics and Radio Society (NERG).

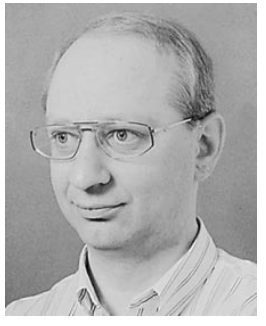

Matti H. A. J. Herben (S'80-M'83-SM'88) was born in Klundert, The Netherlands, in 1953. He received the M.Sc. degree (cum laude) in electrical engineering in 1978 and the Ph.D. degree in technical sciences in 1984, both from Eindhoven University of Technology (EUT), The Netherlands.

Since 1978 he has been employed at the Radiocommunications Group of EUT, currently as an Associate Professor. His research interests and publications are in the areas of design and numerical analysis of reflector and lens antenna systems, radio interference reduction, electromagnetic wave propagation on terrestrial and satellite links, remote sensing of the turbulent troposphere, and microwave radiometry.

Dr. Herben is a member of the Royal Institute of Engineers (Klvl) in The Netherlands, the Netherlands Electronics and Radio Society (NERG), and the Dutch URSI committee. 